\title{
A Hybrid Constraint Programming and Enumeration Approach for Solving NHL Playoff Qualification and Elimination Problems
}

\author{
Tyrel Russell ${ }^{\mathrm{a}, *}$, Peter van Beek ${ }^{\mathrm{a}}$ \\ ${ }^{a}$ Cheriton School of Computer Science, University of Waterloo \\ Waterloo, Ontario, Canada N2L $3 G 1$ \\ \{tcrussel,vanbeek\}@cs.uwaterloo.ca
}

\begin{abstract}
Many sports fans invest a great deal of time into watching and analyzing the performance of their favorite team. However, the tools at their disposal are primarily heuristic or based on folk wisdom. We provide a concrete mechanism for calculating the minimum number of points needed to guarantee a playoff spot and the minimum number of points needed to possibly qualify for a playoff spot in the National Hockey League (NHL). Our approach uses a combination of constraint programming, enumeration, network flows and decomposition to solve the problem efficiently. The technique can successfully be applied to any team at any point of the season to determine how well a team must do to make the playoffs.
\end{abstract}

Keywords: constraint satisfaction, network flows, optimization

\section{Introduction}

As a season progresses, sports fans become intensely focused on the playoff race and the position of their team in the standings. Sports sections of major newspapers publish the results of the games and announce when teams have qualified for the playoffs and when they have been eliminated (e.g., the Globe and Mail). However, the newspapers use a heuristic measure for determining when teams have qualified for or been eliminated from the playoffs and announcements are sometimes not made until several days late. Since fans are interested in knowing

\footnotetext{
${ }^{*}$ Corresponding author
} 
when their team has clinched as early as possible, the exact answer is of more interest as the heuristic answer may not give precise results.

However, if the team has not clinched a playoff spot, the decision version of the problem provides no information about how close a team is to earning a playoff position. The problem of determining how close a team is to clinching a playoff spot can be modeled as an optimization problem that determines the minimum number of points that is necessary to guarantee a spot. This bound on the number of points can also be used to determine when a team has no guarantee of making the playoffs and when a team has lost a crucial game and left destiny in the hands of another team. These bounds give both fans and the managers of the teams additional information. For a coach, there is the additional benefit of knowing which games must be won so they can rest injured players before the playoffs. The qualification and elimination problems addressed here are NP-Hard problems and an efficient solution is not known to exist (McCormick, 1999; Gusfield \& Martel, 2002).

In this paper, we propose a hybrid constraint programming and enumeration method to exactly solve qualification and elimination problems for the National Hockey League (NHL) playoffs ${ }^{1}$. Our solutions to both the qualification and elimination problems use a phased strategy that solves enumerated sub-problems with network flows and constraint programming. Constraint programming is a method for modeling and solving combinatorial problems (see, for example, Rossi et al. (2006) for more details on constraint programming). In constraint programming, instances are usually solved using backtracking search interleaved with constraint propagation, where constraint propagation is a method for enforcing local levels of consistency on the constraints.

We experimentally evaluated our proposed approach using instances from the 2005-06 and 2006-07 seasons. For these seasons, qualification of teams was shown up to five days earlier than the Globe \& Mail (2005-2007). The qualification and elimination versions of the problem can be solved in seconds in most cases and within ten minutes for all instances. In sports, analysts, reporters and coaches often refer to "must win" games. Our proposed method can identify games where, by losing the game, a team puts its playoff aspirations into the hands of its opponents. Nine teams in the 2006-07 season are identified that lost at least one of these "must win" games and found themselves in a position to earn a playoff spot again only through the actions of their opponents.

\footnotetext{
${ }^{1}$ Portions of this work have previously been published in Russell \& van Beek (2008, 2009a,b).
} 


\section{Related Work}

The problem of determining when a sports team has mathematically clinched a playoff spot has been well studied for several sports, including baseball (Schwartz, 1966; Robinson, 1991; Wayne, 2001; Adler et al., 2002) and soccer (Ribeiro \& Urrutia, 2005). The problem is known as a winner determination problem. Schwartz (1966) first looked at this type of problem algorithmically for baseball. Two optimization variants of winner determination problems are discussed in this paper: the playoff qualification problem and the playoff elimination problem.

Approaches for solving these problems have been proposed for the Brazilian football championship (Ribeiro \& Urrutia, 2005) and for Major League Baseball (Adler et al., 2002); both approaches use integer programming. However, these sports have a simpler scoring model-where a scoring model defines the reward for each possible outcome of a game - or a simpler playoff qualification method than hockey. Robinson (1991) gives a model for determining the number of points needed to clinch a playoff spot in the National Basketball Association and solves the model using integer programming techniques. Robinson (1991) also gives a model for the NHL but the model did not allow for wild card teams or tiebreaking. Gusfield \& Martel (2002) use similar methods for calculating bounds on when a team has been eliminated from baseball playoffs. Our work differs from theirs in that there are multiple wild card teams in the NHL. Cheng \& Steffy (2008) study the problem of determining qualification for the NHL using integer programming. However, their model could not be solved when secondary and tertiary tie-breaking rules were included.

Wayne (2001) introduces the concept of a lower bound that could be used to determine whether or not a team was eliminated from the playoffs. Gusfield \& Martel (2002) show how this idea can be extended to include a single wild card team. The existence of an upper bound is discussed in this paper. Kern \& Paulusma (2004) show that the approach by Schwartz (1966) could also be used for other scoring models if the scoring model is normalized. However, Kern and Paulusma make an assumption regarding the play of the distinguished team $t_{q}$ that is infeasible for the optimization problems studied here, as it is assumed $t_{q}$ wins or loses every remaining game, for elimination or qualification, respectively.

\section{The National Hockey League}

The NHL consists of thirty teams evenly arranged into two conferences, East and West. Each conference in turn is composed of three divisions with five teams 
in each division. Every team plays 82 games with 41 home games and 41 away games. Teams play six games against each team in their division, four games against teams in their conference but not their division and one or two games against teams in the opposite conference. An NHL game must end in a win or a loss. Each game consists of regulation time - three periods of twenty minutes each-and, if tied at the end of regulation time, a five minute overtime, which ends when a goal is scored. If the game remains tied after overtime, a shootout is conducted, which must conclude with a winner. The NHL has a unique scoring model. The winner of the game always earns two points. The losing team earns no points if the game ends during regulation time, but earns one point if the game ends during an overtime period or a shootout.

Teams are placed in standings by number of points earned, for both divisional and conference standings. In both conferences, a team makes the playoffs if they are a division leader or one of the top five teams that are not division leaders in their conference. If there are ties in the number of points, the NHL uses three different tie-breaking measures to determine the standings. The first tie-breaking measure is to compare the number of wins by each team. If teams are still tied after comparing the number of wins, the second tie-breaking measure is to compare the number of points earned against only those teams that are tied. The third tiebreaking measure is the total number of goals scored in the entire regular season. In our work, we include the first and second tie-breaking measures, but not the third, as the number of goals in a season cannot be determined or bounded in advance. For qualification, it is assumed that a team has not qualified if they need to win via the third tie breaker. Conversely, for elimination, it is assumed that a team has a chance to qualify if they could win via the third tie breaker.

\section{A Motivating Example}

As an illustrative example that will be used throughout the paper, a simplified six team league is constructed where teams play each other exactly four times, for a total of 20 games each, and only four of the six teams will make the playoffs. For simplicity, divisions are not introduced in this example but are discussed later in the paper. The six teams are Boston, Chicago, Detroit, Montreal, New York and Toronto, and a round robin tournament with four identical rounds is generated. The schedule can be seen in Figure 1. In the example, the first thirteen games of the season have been played and each team has seven games remaining. The symmetry is for clarity and is not required by the techniques, as in practice not every team plays on each game day. From the results of the games, the standings 


\begin{tabular}{|c|c|c|c|c|c|c|}
\hline 30 & 31 & $\begin{array}{l}1 \\
\text { M:0,T:2 } \\
\text { NY:2,B:1 } \\
\text { D:2,C:0 }\end{array}$ & $\begin{array}{l}2 \\
\text { T:2,B:1 } \\
\text { M:1,D:2 } \\
\text { NY:2,C:0 }\end{array}$ & 3 & $\begin{array}{l}4 \\
\text { T:2,NY:0 } \\
\text { M:2,C:0 } \\
\text { B:2,D:1 }\end{array}$ & $\begin{array}{l}5 \\
\mathrm{~T}: 1, \mathrm{C}: 2 \\
\mathrm{M}: 2, \mathrm{~B}: 1 \\
\mathrm{NY}: 0, \mathrm{D}: 2\end{array}$ \\
\hline 6 & $\begin{array}{l}7 \\
\text { T:2,D:0 } \\
\text { NY:2,M:0 } \\
\text { B:1,C:2 }\end{array}$ & $\begin{array}{l}8 \\
\text { M:1,T:2 } \\
\text { NY:2,B:0 } \\
\text { D:1,C:2 }\end{array}$ & 9 & $\begin{array}{l}10 \\
\text { T:0,B:2 } \\
\text { M:2,D:1 } \\
\text { NY:1,C:2 }\end{array}$ & 11 & $\begin{array}{l}12 \\
\text { T:0,NY:2 } \\
\text { M:2,C:0 } \\
\text { B:1,D:2 }\end{array}$ \\
\hline $\begin{array}{l}13 \\
\text { T:0,C:2 } \\
\text { M:2,B:1 } \\
\text { NY:2,D:0 }\end{array}$ & 14 & $\begin{array}{l}15 \\
\text { T:1,D:2 } \\
\text { NY:2,M:0 } \\
\text { B:2,C:0 }\end{array}$ & $\begin{array}{l}16 \\
\text { M:2,T:1 } \\
\text { NY:2,B:0 } \\
\text { D:2,C:0 }\end{array}$ & 17 & $\begin{array}{l}18 \\
\text { T:2,B:0 } \\
\text { M:2,D:0 } \\
\text { NY:2,C:0 }\end{array}$ & $\begin{array}{l}19 \\
\text { T:2,NY:0 } \\
\text { M:2,C:0 } \\
\text { B:2,D:1 }\end{array}$ \\
\hline 20 & $\begin{array}{l}21 \\
T \vee C \\
M \vee B \\
N Y \vee D\end{array}$ & $\begin{array}{l}22 \\
\text { T v D } \\
\text { NY v M } \\
B \text { v C }\end{array}$ & 23 & $\begin{array}{l}24 \\
M \text { v T } \\
N Y \text { v B } \\
D \text { v C } \\
\end{array}$ & 25 & $\begin{array}{l}26 \\
T \vee B \\
M \vee D \\
N Y \text { v C } \\
\end{array}$ \\
\hline $\begin{array}{l}27 \\
T \vee N Y \\
M \vee C \\
B \vee D\end{array}$ & 28 & $\begin{array}{l}29 \\
\text { T v C } \\
M \text { v B } \\
\text { NY v D }\end{array}$ & $\begin{array}{l}30 \\
\text { T v D } \\
\text { NY v M } \\
B \text { v C }\end{array}$ & 1 & 2 & 3 \\
\hline
\end{tabular}

Figure 1: An example schedule for six teams where each team plays each other team four times for a total of sixty of games. Thirteen of each team's games have been played and the results, in terms of points, are noted against the played games. The remaining seven games that each team must still play are listed with their scheduled opponents.

can be constructed (see Table 1) and the games remaining between each pair of teams tabulated (see Table 2).

\section{Basic Models}

In this paper, we solve two related optimization problems: the playoff qualification problem and the playoff elimination problem.

Definition 1 (Playoff Qualification Problem). Given a remaining schedule of games left to play, the results up to a given point of the season-i.e. points and wins earned by teams so far-and a distinguished team $t_{q}$, the playoff qualification problem is to determine the minimum number of points needed by $t_{q}$ such that if they earn that number of points there exists no scenario, i.e. a completion of the remaining games, such that $t_{q}$ does not qualify for the playoffs.

Definition 2 (Playoff Elimination Problem). Given a remaining schedule of games left to play, the results up to a given point of the season-i.e. points and wins earned by teams so far-and a distinguished team $t_{q}$, the playoff elimination problem is to determine the minimum number of points such that if $t_{q}$ earns that number of points there exists at least one scenario where $t_{q}$ earns a playoff spot. 
Table 1: The standings of the hypothetical league after the first thirteen games of the schedule have been played. Each team is awarded 2 points for each win, 0 points for each loss and 1 point for each overtime loss.

\begin{tabular}{c|cc|ccc|c} 
Team & $\begin{array}{c}\text { Games } \\
\text { Played }\end{array}$ & $\begin{array}{c}\text { Games } \\
\text { Remaining }\end{array}$ & Wins & $\begin{array}{c}\text { Losses } \\
\text { Overtime } \\
\text { Losses }\end{array}$ & Points \\
\hline New York & 13 & 7 & 9 & 3 & 1 & 19 \\
Montreal & 13 & 7 & 8 & 3 & 2 & 18 \\
Toronto & 13 & 7 & 7 & 3 & 3 & 17 \\
Detroit & 13 & 7 & 6 & 3 & 4 & 16 \\
\hline Boston & 13 & 7 & 4 & 3 & 6 & 14 \\
Chicago & 13 & 7 & 5 & 8 & 0 & 10
\end{tabular}

The set of teams in the NHL is denoted $T=\left\{t_{1}, \ldots, t_{n}\right\}$. Let $C_{i}$ be the set of indices of teams in the conference and $D_{i}$ be the set of indices of teams in the division to which team $t_{i}$ belongs. Let $T B_{i}$, the tie-breaking set, be the set of indices of the teams tied with team $t_{i}$, including $t_{i}$, in both points and wins from the same conference at the end of the regular season.

We introduce the lower case letters $w, o$ and $p$ to represent the variables for wins, overtime losses and points, respectively, earned at the end of the regular season. We use the upper case letters $W, O, P$ and $G$ to represent the constant values of wins earned, overtime losses earned, points earned and games remaining, respectively, up to the current date of the regular season.

For every team $t_{i}$ and opponent $t_{j}, w_{i j}$ and $o_{i j}$ represent the number of wins and overtime losses earned at the end of the regular season by $t_{i}$ over $t_{j}$ and $W_{i j}$ and $O_{i j}$ represent the number of wins and overtime losses earned up to the current date of the season. The points $p_{i j}$ earned by team $t_{i}$ against opponent $t_{j}$ at the end of the regular season is the weighted sum of the wins, worth two points, and the overtime losses, worth a single point. The total points $p_{i}$ earned at the end of the regular season by team $t_{i}$ is the sum of the points earned against all opponents. Let $G_{i j}$ represent the number of games remaining for $t_{i}$ against $t_{j}$ at the current date and let $G_{i}=\sum_{j} G_{i j}$ represent the number of games remaining for $t_{i}$ against all teams. A scenario $S$ is a completion of the schedule by assigning results to the remaining games. For each team $t_{i}$, let $m p p_{i}=\max _{S}\left(p_{i}\right)$ be the maximum possible points that could be earned by $t_{i}$ under any scenario.

At the conclusion of the regular season, eight teams from each conference for 
Table 2: The number of games remaining for each team against each opponent after the thirteen completed games in the example.

\begin{tabular}{c|cccccc} 
Teams & Boston & Chicago & Detroit & Montreal & New York & Toronto \\
\hline Boston & - & 2 & 1 & 2 & 1 & 1 \\
Chicago & 2 & - & 1 & 1 & 1 & 2 \\
Detroit & 1 & 1 & - & 1 & 2 & 2 \\
Montreal & 2 & 1 & 1 & - & 2 & 1 \\
New York & 1 & 1 & 2 & 2 & - & 1 \\
Toronto & 1 & 2 & 2 & 1 & 1 & -
\end{tabular}

a total of sixteen teams qualify for the playoffs. A team $t_{i}$ qualifies for the playoffs if (a) $t_{i}$ is a division leader; i.e., the team with points greater (or equal with better tie breakers) than all other teams in its division, or (b) $t_{i}$ is a wild card team; i.e., not a division leader but with points greater (or equal with better tie breakers) than at least seven other teams in its conference that are not division leaders. A team has qualified when the points needed to guarantee a playoff spot is zero. A team $t_{i}$ controls their own destiny if the points needed to guarantee a playoff spot does not exceed $m p p_{i}$. A team $t_{i}$ has been eliminated when the points needed to possibly qualify for a playoff spot exceeds $m p p_{i}$.

The basic model of the NHL Qualification Problem is a combination of nine constraints (Constraints (1)-(9) below). Following Ribeiro \& Urrutia (2005), the problem of finding the minimum points necessary for a team $t_{q}$ to qualify is converted into the problem of finding the maximum number of points such that there exists a scenario where $t_{q}$ could have been eliminated. The number of points needed to clinch a playoff spot is then one greater than this maximum. Constraint (1) enforces that each game must end in a winner. Constraint (2) enforces that each team earns only one win or one overtime loss for each game played. Constraint (3) enforces that the total wins is the sum of the wins against all opponents. Constraints (4) and (5) enforce that the points between two teams is the weighted sum of wins and overtime losses and the total points is the sum of points against all opponents, respectively. Constraint (6) formally defines the tie-breaking sets in terms of the model variables. Constraint (7) states that to be better than $t_{q}$ a team must be better on points (7a), better on tie breakers (7b and 7c) or be a division leader (7d). An indicator variable $b_{i}$ is introduced that is 1 if and only if $t_{i}$ has more points or better tie breakers than $t_{q}$ or is a division leader. Constraint (8) 
enforces that there must be at least eight teams in $t_{q}$ 's conference-i.e., playoff contenders - that are better than $t_{q}$. Constraint (9) ensures that $t_{q}$ is not a division leader by enforcing that there must exist some team $t_{i}$, different from $t_{q}$, that is the leader of $t_{q}$ 's division.

$$
\begin{aligned}
& \max p_{q}, \\
& \forall_{i, j \in T} \quad w_{i j}+w_{j i}=W_{i j}+W_{j i}+G_{i j}, \\
& \forall_{i, j \in T} \quad w_{i j}+o_{i j} \leq W_{i j}+O_{i j}+G_{i j} \\
& \forall_{i \in T} \quad w_{i}=\sum_{j \in T} w_{i j}, \\
& \forall_{i, j \in T} \quad p_{i j}=2 w_{i j}+o_{i j}, \\
& \forall_{i \in T} \quad p_{i}=\sum_{j \in T} p_{i j}, \\
& \forall_{i, j \in T} \quad\left(j \in T B_{i}\right) \Leftrightarrow\left(p_{i}=p_{j} \wedge w_{i}=w_{j} \wedge j \in C_{i}\right), \\
& \forall_{i \in C_{q}} \quad b_{i}=1 \\
& \Leftrightarrow \quad\left(p_{i}>p_{q}\right) \\
& \vee \quad\left(p_{i}=p_{q} \wedge w_{i}>w_{q}\right) \\
& \vee \quad\left(p_{i}=p_{q} \wedge w_{i}=w_{q} \wedge \sum_{j \in T B_{q}} p_{i j}>\sum_{j \in T B_{q}} p_{q j}\right) \\
& \vee \quad\left[\forall_{d \in D_{i}}\left(p_{i}>p_{d}\right) \vee\left(p_{i}=p_{d} \wedge w_{i}>w_{d}\right)\right. \\
& \left.\vee\left(p_{i}=p_{d} \wedge w_{i}=w_{d} \wedge \sum_{j \in T B_{i} \cap D_{i}} p_{i j}>\sum_{j \in T B_{i} \cap D_{i}} p_{d j}\right)\right], \\
& \sum_{i \in C_{q}} b_{i} \geq 8 \\
& \exists_{i \in D_{q}} \quad i \neq q \wedge\left[\forall_{d \in D_{q}}\left(p_{i}>p_{d}\right) \vee\left(p_{i}=p_{d} \wedge w_{i}>w_{d}\right)\right. \\
& \left.\vee\left(p_{i}=p_{d} \wedge w_{i}=w_{d} \wedge \sum_{j \in T B_{i} \cap D_{q}} p_{i j}>\sum_{j \in T B_{i} \cap D_{q}} p_{d j}\right)\right] .
\end{aligned}
$$


The NHL Elimination Problem has a similar model where the solution is the minimum number of points such that $t_{q}$, the team under consideration, could do no worse and still make the playoffs.

$$
\begin{aligned}
& \min p_{q}, \\
& \forall_{i, j \in T} \quad w_{i j}+w_{j i}=W_{i j}+W_{j i}+G_{i j}, \\
& \forall_{i, j \in T} \quad w_{i j}+o_{i j} \leq W_{i j}+O_{i j}+G_{i j}, \\
& \forall_{i \in T} \quad w_{i}=\sum_{j \in T} w_{i j}, \\
& \forall_{i, j \in T} \quad p_{i j}=2 w_{i j}+o_{i j}, \\
& \forall_{i \in T} \quad p_{i}=\sum_{j \in T} p_{i j} \\
& \forall_{i, j \in T} \quad\left(j \in T B_{i}\right) \Leftrightarrow\left(p_{i}=p_{j} \wedge w_{i}=w_{j} \wedge j \in C_{i}\right), \\
& \forall_{i \in C_{q}} \quad \omega_{i}=1 \\
& \Leftrightarrow \quad\left[\left(p_{i}<p_{q}\right)\right. \\
& \vee \quad\left(p_{i}=p_{q} \wedge w_{i}<w_{q}\right) \\
& \left.\vee \quad\left(p_{i}=p_{q} \wedge w_{i}=w_{q} \wedge \sum_{j \in T B_{q}} p_{i j} \leq \sum_{j \in T B_{q}} p_{q j}\right)\right] \\
& \wedge \quad\left[\exists_{d \in D_{i}}\left(p_{i}<p_{d}\right) \vee\left(p_{i}=p_{d} \wedge w_{i}<w_{d}\right)\right. \\
& \left.\vee\left(p_{i}=p_{d} \wedge w_{i}=w_{d} \wedge \sum_{j \in T B_{i} \cap D_{i}} p_{i j}<\sum_{j \in T B_{i} \cap D_{i}} p_{d j}\right)\right], \\
& \left(\sum_{i \in C_{q}} \omega_{i} \geq 7\right) \vee\left[\forall_{d \in D_{q}}\left(p_{q}>p_{d}\right) \vee\left(p_{q}=p_{d} \wedge w_{q}>w_{d}\right)\right. \\
& \left.\vee\left(p_{q}=p_{d} \wedge w_{q}=w_{d} \wedge \sum_{j \in T B_{q} \cap D_{q}} p_{q j}>\sum_{j \in T B_{q} \cap D_{q}} p_{d j}\right)\right] .
\end{aligned}
$$

Constraints (10)-(15) in the NHL Elimination Problem are identical to Constraints (1)-(6) in the NHL Qualification Problem. Constraint (16) states that a team is 
worse than $t_{q}$ if they have less points (16a) or equal points and worse tie breakers than $t_{q}(16 \mathrm{~b}$ or $16 \mathrm{c})$ and the team is not a division leader (16d). Constraint (17) states that $t_{q}$ qualifies for the playoffs if there are seven teams worse than $t_{q}$ or $t_{q}$ is a division leader.

Both of the qualification and elimination models have 1800 integer variables with domains in $[0 \ldots 8]\left(w_{i j}\right.$ and $\left.o_{i j}\right), 900$ variables with domains in $[0 \ldots 16]$ $\left(p_{i j}\right)$, 30 variables with domains $[0 \ldots 164]\left(p_{i}\right)$ and 15 variables with $[0,1]$ domains $\left(b_{i}\right.$ or $\left.\omega_{i}\right)$. The qualification model contains 3647 constraints and the elimination model contains one less.

\section{Solving the Model}

\subsection{Overview}

In this section, we give an overview of the solvers for qualification and elimination problems. The solver for the qualification problem uses a phased approach that decomposes the problem based on Constraint (7). The approach here, of enumerating and using different phases, differs from (Van Hentenryck, 1989, pg. 169) as it is possible to determine when a problem is hard and can be reserved until later, if needed. In Constraint (7), the first three conditions (7a, 7b, 7c) form a tie-breaking constraint. These three conditions have a mutually exclusive structure as a relaxed version of (7a), where equality is allowed between $p_{i}$ and $p_{q}$, would include all solutions to (7b) and (7c), and a relaxed version of (7b), where equality is allowed between $w_{i}$ and $w_{q}$, would include all solutions to (7c).

The first phase of the solver enforces (7a) but not (7b) and (7c). Using enumeration, the maximum number of points earned by any sufficient set of better teams gives a bound $\mathbf{p}$ on the number of points $t_{q}$ would need to earn. If the bound $\mathbf{p}$ is less than $P_{q}$, the points earned by $t_{q}$ so far in the season, $t_{q}$ has guaranteed a playoff spot. If the bound $\mathbf{p}$ is more than $m p p_{q}$, the maximum possible points that could be earned by $t_{q}$ at the end of the regular season, $t_{q}$ cannot guarantee a playoff spot. In all other situations, further tie-breaking is necessary and $\mathbf{p}$ and the sets with point value $\mathbf{p}$ are passed to the second phase of the solver.

The second phase of the solver enforces (7a) and (7b) but not (7c). Given the sets from the first phase and the point bound $\mathbf{p}$, each set is checked to determine if there exists a scenario where each team in the set could earn more than p points or exactly $\mathbf{p}$ points and more wins when $t_{q}$ has $\mathbf{p}$ points. If such a scenario exists, $t_{q}$ would need one more point to guarantee a playoff position. If $t_{q}$ cannot earn that extra point, i.e. $\mathbf{p}+1>m p p_{q}, t_{q}$ cannot guarantee a playoff spot. If no such scenario exists, all of the sets where teams could earn more points or equal 
points and equal or more wins are found. If no such set of teams exists, $t_{q}$ would guarantee with p points. If such sets of teams do exist, further tie-breaking is necessary and the sets are passed to the third phase of the solver.

The third phase solves a decision version of the complete model. If there exists a solution, $t_{q}$ would need $\mathbf{p}+1$ points to guarantee. As before, if $\mathbf{p}+1>m p p_{q}, t_{q}$ cannot guarantee a playoff spot. If there does not exist a solution, $t_{q}$ would need p points to guarantee a playoff spot.

Much of the same mechanisms can be used for the playoff elimination problem. The goal is to search for any set of seven teams to occupy the spots below $t_{q}$. If this set of teams exist or if $t_{q}$ is a division leader at a given point bound then $t_{q}$ can possibly qualify for the playoffs. Using a similar bounding technique, a tight bound can be obtained and the same phased solving methods can be applied.

We next describe each of the three phases in more detail.

\subsection{The First Phase}

In this section, the mechanisms for solving the first phase of the solver are described. Identifying sets of teams that could potentially be division leaders and wild card teams is presented as a technique for relaxing the constraints of the model. Combined with the enumeration of these sets, a bound is generated and the bounding of the sets provides a tight lower bound on the actual value of the complete problem. Given the enumeration and bounding, it is simple to answer the two necessary questions for the first phase: If the bound $\mathbf{p}$ is less than $P_{q}$ then $t_{q}$ has already qualified and if $\mathbf{p}$ is greater than $m p p_{q}$ then guaranteeing qualification is not possible.

\subsubsection{Enumerating the set of implication constraints}

Constraint (8) requires that there be at least eight teams where Constraint (7) is satisfied (by enforcing that at least eight of the indicator variables are true). It is possible to enumerate, in the case of NHL teams, all of the sets of enforced indicators possible under (8).

An Elimination Set, $E$, is a set of eight or more teams from the same conference that includes at least one team from each division and does not include $t_{q}$. Each team $t_{i} \in E$ must either have $m p p_{i}>P_{q}$ or be the only team in $E$ from a division $D_{i}$ such that $D_{i} \neq D_{q}$. We restrict the size of the sets considered to those that contain exactly eight teams as any solution with more teams is also a solution

with only eight teams. The intuition is that an elimination set represents a possible set of teams that would all make the playoffs and so eliminate $t_{q}$. 
Example 1 (Finding Elimination Sets). Our example league (see Section 4) has four playoff positions. Hence, the elimination set has four teams. Given $t_{q}=$ New York, the elimination sets are formed from the remaining five teams. Every subset of size four that can earn more points than the number of points New York currently has is a viable elimination set. The elimination sets are:

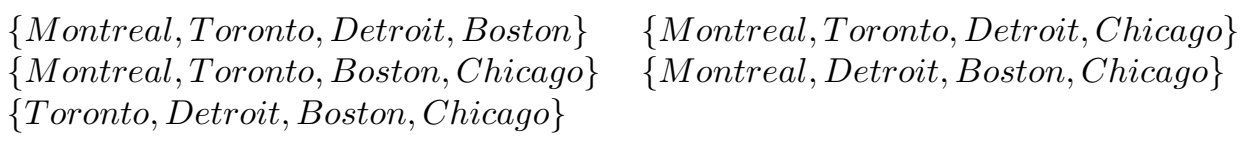

A Qualification Set, $Q$, is a set of seven or more teams from the same conference that does not include any team that has clinched a division leadership position and does not include $t_{q}$. Only sets with the smallest number of teams, seven, are considered because a solution with more teams is also a solution for seven teams. The intuition is that a qualification set is a possible set of teams that would not make the playoffs if $t_{q}$ qualified for the playoffs.

Example 2 (Finding Qualification Sets). Our example league has six teams and four playoff spots. Therefore, a qualification set would have two teams. The only pairs in this example that are not qualification sets are the ones including New York. The qualification sets are:

$$
\begin{array}{llll}
\{\text { Montreal }, \text { Toronto }\} & \{\text { Boston, Chicago }\} & \{\text { Montreal }, \text { Detroit }\} & \text { Detroit, Boston }\} \\
\{\text { Montreal, Chicago }\} & \{\text { Toronto, Boston }\} & \{\text { Montreal, Boston }\} & \\
\{\text { Toronto, Chicago }\} & \{\text { Toronto, Detroit }\} & \{\text { Detroit }, \text { Chicago }\} &
\end{array}
$$

Elimination (and qualification) sets are used to simplify the basic model. Since we know exactly which teams must be better (or worse), Constraint (8) can be removed as it will always be satisfied and Constraint (7) is replaced by,

$$
\forall i \in E\left(p_{i}>p_{q}\right) \vee\left(p_{i}=p_{q} \wedge w_{i}>w_{q}\right) \vee \ldots .
$$

However, instead of a single model, the problem must now be solved for each enumerated elimination set and the best solution is taken.

\subsubsection{Calculating the bound}

Since the NHL Playoff Qualification Problem is an optimization problem, not all of the feasible solutions lead to an optimal solution and bounding can be used to remove feasible solutions that are non-optimal. The bound of an elimination set $E$ is given by $\max _{S}\left(\min _{i \in E}\left(p_{i}\right)\right)$, where $S$ is the set of scenarios and $p_{q}=\min \left(\min _{i \in E}\left(p_{i}\right), m p p_{q}\right)$. The bound on the qualification set 
$Q$ is given by $\min _{S}\left(\max _{i \in Q}\left(p_{i}\right)\right)$, where $S$ is the set of scenarios and $p_{q}=$ $\max \left(\max _{i \in Q}\left(p_{i}\right), P_{q}\right)$.

Given an elimination set $E$, the bound is calculated by relaxing the model described at the end of Section 6.2.1 replacing Constraint (18) with,

$$
\forall_{i \in E}\left(p_{i} \geq p_{q}\right) .
$$

Constraint (19) is a valid relaxation as it contains all possible solutions to Constraint (18). We now show that this model can be solved using a sequence of network flow calculations. First, some points can be determined initially,

$$
p_{i}^{\prime}=P_{i}+2 \sum_{j \notin C_{i}} G_{i j}+2 \sum_{j \notin E \cup\left\{t_{q}\right\}} G_{i j}+\sum_{j \in E \cup\left\{t_{q}\right\}} G_{i j} .
$$

Equation 20 represents the sum of the points already earned $\left(P_{i}\right)$, the wins against teams not in the set $E \cup\left\{t_{q}\right\}\left(2 \sum_{j \notin C_{i}} G_{i j}+2 \sum_{j \notin E \cup\left\{t_{q}\right\}} G_{i j}\right)$ and one point each from games against teams in $E \cup\left\{t_{q}\right\}\left(\sum_{j \in E \cup\left\{t_{q}\right\}} G_{i j}\right)$. These points are valid because every increase does not affect the maximum possible points of the other teams in $E$.

We want to find a point value where there is a feasible flow on a specifically constructed flow graph. The first step is to find an initial value for the bounding procedure determined by relaxing the constraints that a specific number of games must be played between two teams and that $t_{q}$ must be able to earn that many points. First, the lowest $m p p_{i}$ for all teams $i \in E$ is found. Next, the points described by Equation 20 are calculated for each team and any points that could be earned by playing $t_{q}$ are added. During this step, a count of the number of remaining games is kept. The teams are sorted by increasing point values. Games from the count of remaining games are added to the worst or set of equally worst teams until the lowest $m p p_{i}$ is reached or the count is reduced to zero. Lemma 1 shows that the initial value is greater than the actual bound.

Lemma 1 (The Initial Value). The initial value is greater than the actual bound.

Proof. Proof by contradiction. Assume that there exists an actual bound that is higher than the initial value given by the algorithm. This assumes that there exists some assignment of wins that is not possible under the relaxations. Since two constraints are removed and none are added, the relaxation must be valid. Therefore, the solution to the original problem must be contained in the set of solutions to the relaxation and any bound achievable on the original problem is achievable under the relaxation. 


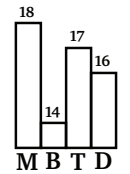

(a)

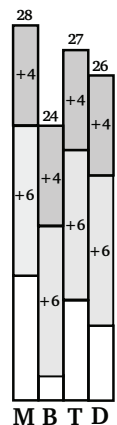

(b)

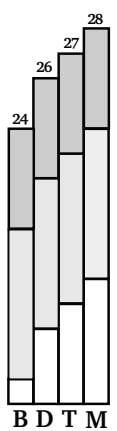

(c)

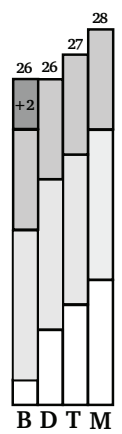

(d)

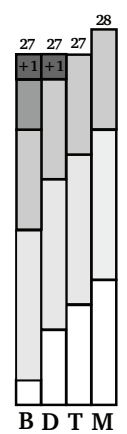

(e)

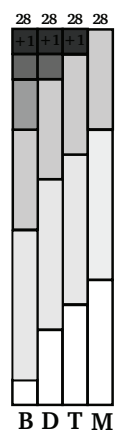

(f)

Figure 2: The initial value calculation for the example. (a) The number of points earned by each team. (b) Added six points for each team for the three games they play against both New York and Chicago and four points for the four remaining games. (c) The sorted list of teams. (d) Two wins are added to Boston. (e) A single point each is added to Boston and Detroit. (f) Three points are added to the three weakest teams and the algorithm terminates with a single game remaining.

Example 3 (Calculating the Initial Value). Referring to the example described in Section 4, the first elimination set described previously in Example 1 is Montreal, Toronto, Detroit and Boston. This leaves New York and Chicago out of the set. Boston has the lowest maximum possible points, where $m p p_{B}=28$. Figure $2 a$ shows the current points of the teams (See Table 1). Figure $2 b$ shows the points, two per game, added for the games outside the set and the points, one point per game, added for the games inside the set (See Table 2). Teams are sorted in ascending order as shown in Figure 2c. Boston has two less points than Detroit so two of the remaining games are assigned to Boston (See Figure 2d). One game is added to Boston and Detroit (See Figure 2e). Boston, Chicago and Toronto each have one point less than Montreal and one game each is assigned (See Figure $2 f$ ). The lowest $m p p_{i}$ has been reached, so the algorithm returns 28 . All other elimination sets would have a bound of 24 because Chicago can earn at most 24 points. Each initial value must be tightened and made feasible by adding the removed constraints.

The initial value is likely to be infeasible due to the relaxation of necessary constraints. To find a feasible value, a network flow based algorithm is used. Starting with the initial value, we decrease the value until the current value $c$ is feasible. Given a value $c$ every team in the elimination set plus $t_{q}$ needs to win a certain number of games to reach the value. The need $n_{i}$ of a team $t_{i}$ is the 
difference between $c$ and the adjusted points of $t_{i}$,

$$
n_{i}=\max \left(c-p_{i}^{\prime}, 0\right),
$$

where $p_{i}^{\prime}$ is defined in Equation 20.

Once the needs are known for a given elimination set, a source node $s$ and a sink node $t$ are created. A node for every matchup of teams from the set composed of the elimination set plus $t_{q}$ is added and, in a second column, a node for each team in the elimination set plus $t_{q}$. There is an arc from $s$ to every matchup $i, j$ with an upper and lower bound of $G_{i j}$. For a matchup between $t_{i}, t_{j}$, there are two outgoing edges to $t_{i}$ and $t_{j}$ with lower bounds of 0 and upper bounds of $G_{i j}$. For a team $t_{i}$, there is an edge to the sink with a lower bound of $n_{i}$ and an upper bound of $G_{i}$. For $t_{q}$, the edge to the sink has an upper and lower bound of $n_{i}$. An example of such a network can be found in Figure 3.

Example 4 (Calculating the Bound). Referring to the example described in Section 4, the bounding technique is applied to the first set of teams generated in Example 1. In Example 3, the upper bound for this set is 28. The needs are calculated for each team and the flow network in Figure 3 is constructed. The two columns of nodes are the matchups and the teams, respectively. Edges are added to the graph connecting the layers. For example, New York plays Montreal twice so the edge from the source to the New York-Montreal has a capacity of two. From New York-Montreal, there is an edge to New York and an edge to Montreal and both of them have an upper bound of 2 and a lower bound of 0 . New York reaches the bound exactly so its upper and lower bound capacities are equal. Montreal, alternatively, has a need of two but an upper bound of six. Once constructed, a feasible flow algorithm is applied (Ahuja et al., 1993). Figure 3 shows that there exists a feasible flow and the initial value is, in this case, a feasible bound. The bound on all of the other elimination sets is 24.

Constraint (19) gives the relaxed form of the original constraint where only the first tie-breaking condition is considered. Recall that in the basic model we want the maximum number of points where $p_{q}$ is still eliminated. A relaxation of this model gives us an upper bound on the basic model but it turns out that this upper bound is a tight lower bound on the original problem which is to find the minimum number of points such that $p_{q}$ guarantees a playoff spots.

Lemma 2 (One More Point Lemma). Given the set of bounds calculated for each enumerated elimination set, the largest bound in the set is at most one less than the optimal solution to the original problem. 


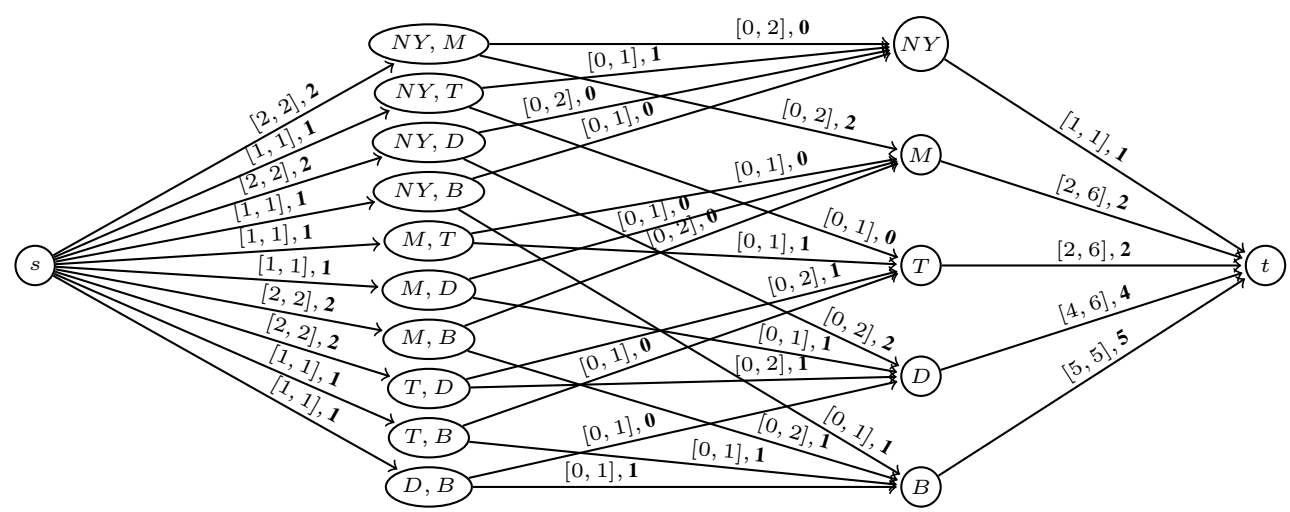

Figure 3: The feasible flow network from Example 4, which shows the elimination set of Montreal, Toronto, Detroit and Boston for New York with a point bound of 28. The arcs are annotated with their upper and lower bound capacities. The bold number represents a possible feasible flow on the network.

Proof. Let $E^{\prime}$ be the elimination set with the highest bound, $\mathbf{p}$. In the case where the bound is greater than $m p p_{q}$, then there is always a solution where $t_{q}$ can be eliminated. Otherwise, the solution obtained is the maximum value of $p_{q}$ such that each team $t_{i}, i \in E^{\prime}$ has at least as many points as $t_{q}$. If $t_{q}$ earns one more point then there must always exist one team $t_{i}, i \in E^{\prime}$ such that $t_{i}$ cannot simultaneously obtain $\mathbf{p}+1$ points along with $t_{q}$ or the original bound was not the maximum solution to the relaxed model. Therefore, if $t_{q}$ earns one more point than the relaxed bound, they guarantee qualification and they could qualify with $\mathbf{p}$ if they are better on tie breaks.

Example 5 (The First Phase). In Example 4, it is shown that there exists a solution to the problem with equality constraints for New York at a point bound of 28 and the set $\{$ Montreal,Toronto, Detroit,Boston\} is the only possible set with an optimal solution as the other sets have a point bound of 24. Since 28 is neither greater than the maximum possible points of New York or less than the current points of New York, no decision can be made and the second phase is needed.

\subsection{The Second Phase}

In the second phase, only the elimination sets where teams can reach the point bound $\mathbf{p}$ are kept from the first phase and, for each set, there exists no scenario where every team in the set earns more points than the bound. We call such sets retained elimination sets. The second phase is looking for sets where every team 
earns more points than the bound or if a team just reaches the bound, the tied team earns more wins. Team $t_{q}$ has a certain number of earned wins, $W_{q}$, and some maximum and minimum possible given $\mathbf{p}$. The win range is defined as,

$$
W_{q}+\max \left(\left(\mathbf{p}-P_{q}\right)-G_{q}, 0\right) \leq w_{q} \leq W_{q}+\left\lfloor\frac{\left(\mathbf{p}-P_{i}\right)}{2}\right\rfloor .
$$

Given the range of win values, each value is checked while bounding the wins of $t_{q}$, denoted $\mathbf{w}$. For each retained elimination set, it is determined whether the teams can collectively exceed $\mathbf{p}$ or $\mathbf{p}$ and exceed $\mathbf{w}$. The difference between the bound and points of a team $\left(\mathbf{p}-P_{i}\right)$ can be reduced by assuming every loss happens in overtime giving each team $G_{i}$ extra points. Therefore, the number of wins needed to reach $\mathbf{p}$ is equal to $\left(\mathbf{p}-P_{i}\right)-G_{i}$. These problems are solved similarly to the previous bound calculation but with adjusted needs. There are four different cases that must be considered: some teams have exceeded $\mathbf{p}$, some teams may be able to reach $\mathbf{p}$ but would have more than $\mathbf{w}$ wins, some teams may reach $\mathbf{p}$ but require more wins to ensure that $\mathbf{w}$ is exceeded and $t_{q}$ which must earn exactly $\mathbf{w}$ wins. The needs and conditions are formalized as follows,

$$
n_{i}= \begin{cases}0 & \text { if }\left(\mathbf{p}-P_{i}\right)-G_{i}<0 \\ \left(\mathbf{p}-P_{i}\right)-G_{i} & \text { if }\left(\mathbf{p}-P_{i}\right)-G_{i}+W_{i}>\mathbf{w} \\ \left(\mathbf{p}-P_{i}\right)-G_{i}+1 & \text { if }\left(\mathbf{p}-P_{i}\right)-G_{i}+W_{i} \leq \mathbf{w} \\ \mathbf{w}-W_{i} & \text { if }\left(p_{i}=\mathbf{p}\right) \wedge\left(w_{i}=\mathbf{w}\right)\end{cases}
$$

Given the needs from Equation 23, this problem is formulated as a feasible flow problem as described in Section 6.2.2 except with new needs. If there exists a solution then $t_{q}$ would need an extra point beyond $\mathbf{p}$ in order to guarantee a playoff spot. If $\mathbf{p}+1$ is greater than $m p p_{q}$ then $t_{q}$ cannot guarantee qualification. Otherwise, $\mathbf{p}+1$ points is returned. If there are no solutions, it must be determined if every team can be at least tied with $\mathbf{p}$ and $\mathbf{w}$. Equation 23 is modified to combine the third condition with the second condition. If there is again no solution, there is no scenario where $t_{q}$ does not qualify when earning $\mathbf{p}$ points and that value is returned. Any sets that have a solution must be passed to the third phase.

Example 6 (The Second Phase). Referring to the example described in Section 4 and last expanded in Example 5, the tie-breaking methodology is expanded. First, using Equation 22, New York must have between 11 and 13 wins if they earn 28 points. Teams need extra wins to reach $\boldsymbol{p}$ so the first criterion of Equation 23 does not hold. For the second criterion, it must be determined how many wins a team absolutely must earn to reach the bound. 


$$
\begin{array}{llll}
\text { Montreal: } & (28-18-7)+8=11 & \text { Detroit: } & (28-16-7)+6=11 \\
\text { Toronto: } & (28-17-7)+7=11 & \text { Boston: } & (28-14-7)+4=11
\end{array}
$$

None of the teams can exceed even the lowest win value without earning another win and the third criteria from Equation 23 is enforced for all teams and each team's need increases by one. Recall, however, from Example 5, that Boston can not increase their need as Boston requires all of their seven games just to reach 28 points. Since Boston cannot earn more than 11 wins, win values of 12 and 13 can be pruned. The previous equation shows that the teams could be tied with 28 points and 11 wins. Thus, the only remaining set has a point bound of 28 , the only eligible win bound is 11 and the third phase is required.

\subsection{The Third Phase}

The third phase of tie-breaking incorporates the most complicated of the tiebreaking constraints. It states that teams who are tied in terms of both points and wins are compared in terms of the number of points earned only against those teams who have also earned exactly that number of points and wins. The tiebreaking sets are enumerated in this phase to allow more efficient propagation. The tie-breaking set $T B_{q}$ is the set of teams tied with $t_{q}$ such that, for every $t_{i} \in T B_{q}, p_{i}=\mathbf{p}$ and $w_{i}=\mathbf{w}$ where $p_{q}=\mathbf{p}$ and $w_{q}=\mathbf{w}$.

The possible membership of a tie-breaking set can be pruned using a similar mechanism to the one introduced in the second phase. The difference is that, unlike the second phase where the fourth criteria of Equation 23 only applied to $t_{q}$, here any team in $T B_{q}$ will compute their need using this criteria. Any possible tie-breaking set where there is no feasible flow can be pruned. The equality modification to Equation 23 from Section 6.3 would also be applied.

Once the tie-breaking sets have been pruned, a feasible solution to Constraint (7) must be found. A constraint programming solver is used to determine the feasibility of the final constraint. The basic model is extended to take advantage of the enumeration from the previous phases. First, the optimization criterion can be removed as the tight bounding has turned the problem into a decision problem. Second, the complicated disjunction (7) is replaced with a series of simpler constraints. The teams which are members of the tie-breaking set must have exactly $\mathbf{p}$ points and $\mathbf{w}$ wins. Additionally, those tie-breaking set members who are also members of the elimination set must have more points against other tie-breaking members than $t_{q}$ while those who are not members of the elimination set do not have to enforce this. If a team is neither a member of the elimination set nor the tie-breaking set then all constraints can be relaxed as those teams do not have to meet any bounds. Those teams who belong to the elimination set but not the 
tie-breaking set have to earn more points or equal points and more wins than $t_{q}$. However, to earn enough points to exceed the bounds, it is only necessary to enforce that the teams reach their need values. By enforcing the needs, the teams necessarily satisfy this constraint.

Example 7 (The Third Phase). Referring to the example described in Section 4 and last expanded in Example 6, recall that there is a single eligible elimination set with $\boldsymbol{p}=28$ and $\boldsymbol{w}=11$. Since Chicago could only earn 24 points, Chicago is not a candidate for the tie-breaking set. Now, the set of New York and Boston combined with any subset of the remaining teams is a valid tie-breaking set. However, recall from Example 6 that each team needs to earn at least one more win (and thus point) in order to not be tied. Observe from Figure 3, that none of the teams exceeded their minimum capacity and no further points could be earned by any team. Therefore, the only valid tie-breaking set is the one that contains the entire set and New York. Now all that remains to be shown is that the constraint program described by the elimination set, point bound, win bound and tie-breaking set has a solution. In fact, there is no solution where this is true. New York can earn at most seven points against Chicago and Montreal must earn eight points against Chicago as there was no slack in the feasible flow to allow Montreal or New York to drop games from teams outside the set. Therefore, New York has 21 points against teams in the set and Montreal only has 20 points against teams within the set and, therefore, New York needs 28 points to guarantee a playoff position.

\subsection{A Note About Division Leaders}

An intentional omission from the preceding discussion of the solution is the issue of division leaders and their special status in sports leagues. A division leader is guaranteed a playoff spot regardless of how it would have actually ranked had it not been at the top of its division. The complication of these division leaders arises from the tie-breaking constraints used. First, it is shown that at most one division leader could be weaker than $t_{q}$.

Lemma 3 (At Most One Weak Division Leader). At most one of the three division leaders can have fewer points or equal points and fewer wins than $t_{q}$ if $t_{q}$ does not clinch a playoff spot.

Proof. Suppose that the two other division leaders have fewer points or equal points and fewer wins than $t_{q}$. This means that every other team in both divisions will have fewer points or equal points and fewer wins than $t_{q}$. Since at most four other teams from $t_{q}$ 's division plus the two division leaders would qualify, $t_{q}$ must 
qualify in seventh at worst. Therefore, at most one division leader can have fewer points or equal points and fewer wins if $t_{q}$ does not qualify for the playoffs.

If a team is a division leader that has fewer points or equal points and fewer wins then there are obviously no other teams that could be in the elimination set. Therefore, any elimination set that contains only a single team from a division that does not include $t_{q}$ can simply drop that team. If the set contains no members from a division, again not containing $t_{q}$, eight sets of seven are generated by leaving out one team.

The more complicated case is when every member of the elimination set from a particular division is also in the tie-breaking set. In this case, it is possible for a team $t_{i}$ to fail to be better than $t_{q}$ but to still also be the division leader. The reason for the discrepancy is that when $t_{i}$ is compared using the third tie-breaking criteria, it is possible for $t_{i}$ to lose the tie break against $t_{q}$ but not the division members. If this pertains to a division not containing $t_{q}$, the problem is solved normally and, if a solution is returned, a valid solution is obtained. If no solution is found or if $t_{q}$ is possibly a division leader, further steps must be taken. Given there is only a small number of possible division leaders, the problem is solved by checking each different division leader.

\subsection{The Elimination Problem}

The focus of the explanation has been on the solver for the qualification problem but the basic mechanics are applied in the same manner for the NHL Playoff Elimination Problem. The major difference is how the point bound is calculated given a qualification set. Instead of finding the maximum point value of the weakest team in the set, the minimum possible point value of the strongest team is found using the feasible flow algorithm. Once the point bound is established, the other major difference is that if a solution is found then the point bound is returned and if there is no solution then the point bound plus one is returned.

Another difference with elimination problems is with division leaders. It must be checked first if $t_{q}$ could be a division leader for fewer points. Note this can be integrated seamlessly with the normal procedure by adding the set containing only the other division members. If this set has a better point value, it is kept and, if not, it is pruned like other sets.

\section{Experimental Results}

The solver was implemented in C++ using the Boost Graph Library (Siek et al., 2001) for the feasible flow calculations and ILOG Solver (ILOG S.A., 1998) 
Table 3: The results of qualification compared against the published Globe and Mail results. Only results that differ are shown.

\begin{tabular}{l|lcc|lcc} 
& \multicolumn{3}{|c|}{ West } & \multicolumn{3}{c}{ East } \\
\hline & Team & Optimal & G \& M & Team & Optimal & G\& M \\
\hline & Dallas & Mar 31 & Apr 1-3 & Ottawa & Mar 22 & Mar 27 \\
& Calgary & Apr 8 & Apr 9-10 & Buffalo & Apr 4 & Apr 5 \\
2005-06 & Nashville & Apr 9 & Apr 10 & New Jersey & Apr 12 & Apr 14 \\
& San Jose & Apr 13 & Apr 14 & Montreal & Apr 14 & Apr 18 \\
& Colorado & Apr 13 & Apr 15 & & & \\
\hline \hline 2006-07 & Nashville & Mar 23 & Mar 24 & Buffalo & Mar 18 & Mar 22 \\
& Detroit & Mar 24 & Mar 25-26 & Atlanta & Apr 2 & Apr 4
\end{tabular}

to solve the final constraint model. The calculated results are compared against those shown in the Globe \& Mail (2005-2007) for the 2005-06 and 2006-07 season. The 2006-07 season results are used to calculate the minimum points needed to clinch a playoff spot. In total, determining the bound for all 30 teams on all 181 game days of the 2006-07 NHL season (5430 problems) took a little over 46 hours on a Pentium 4 PC. Each instance, representing a team at a given date, took less than ten minutes to calculate the bound and those problems near the end of the regular season, where the results matter the most, were calculated in seconds. To verify the basic model is infeasible, we implemented the model in Gecode 3.5.0 (Schulte et al., 2011) but found that $88 \%$ of the qualification instances and $82 \%$ of the elimination instances timed out after an hour.

Each of the instances for the 2005-06 and 2006-07 seasons was tested and the date of qualification for each of the teams was determined. For most instances, the solving time was a fraction of a second. Compared to the results posted in the Globe and Mail, the exact results generated show qualification earlier for nine teams during the 2005-06 season and for four teams during the 2006-07 season (see Table 3). Entries with multiple dates are due to the absence of Sunday editions or unreported results. Qualification was shown earlier than the Globe and Mail by as much as five and four days for the 2005-06 and 2006-07 seasons, respectively.

The bounds are plotted against both the current points of the team and maximum possible points of the team. Figure 4 shows the result calculated for Toronto and Pittsburgh. If the points needed to guarantee are greater than the maximum possible points, the team is no longer able to guarantee a playoff spot and, if the points needed to possibly qualify are greater than the maximum possible points, 


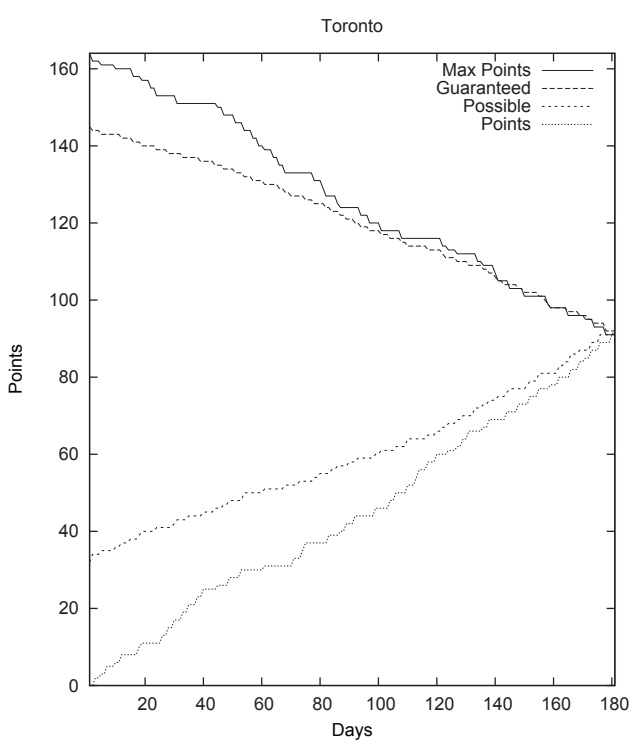

(a)

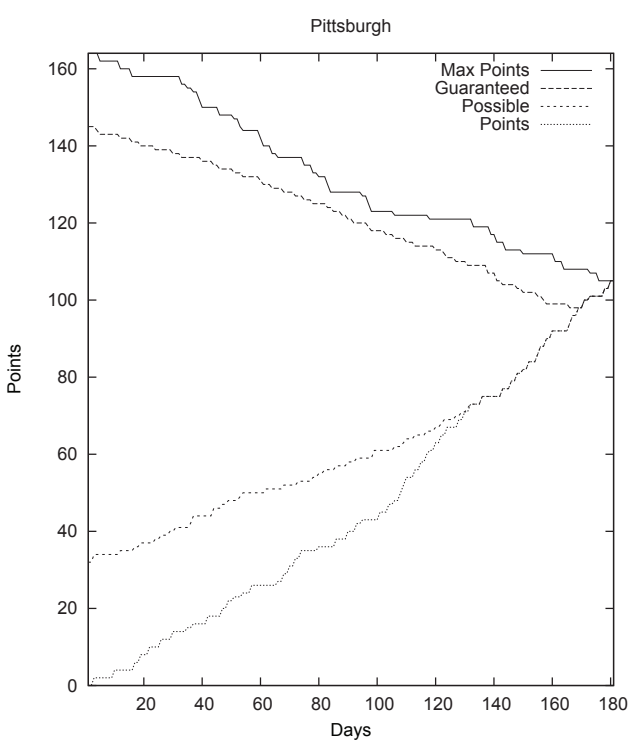

(b)

Figure 4: (a) and (b) The minimum number of points needed by Toronto and Pittsburgh to guarantee or possibly qualify for a playoff spot in the 2006-07 NHL season.

the team is eliminated. A team has qualified if their points are equal to the points needed to guarantee. Toronto placed themselves in a position where guaranteeing a playoff spot was not possible and got lucky four times but did not make the playoffs because the current points never reached the upper bound value.

Table 4 shows the results of the 2006-07 NHL season in terms of the minimum points needed to guarantee a playoff spot. One interesting observation that can be made from this table is that of the nine teams that got a second chance only two of those teams ended up earning a playoff spot.

Table 5 shows the results breakdown of the solver in terms of its phases. The first phase solves 1212 of the 5430 of the problems and in the second phase, with tie-breaking on wins, a further 3773 problems are solved. However, the remaining $8 \%$ of problems require the third phase to break ties. In $47 \%$ of the total instances, the answer differs from the initial lower bound.

\section{Conclusion}

As the season winds down, the fans of the NHL are interested in knowing how far their team is from clinching a playoff spot. A method for solving the 
Table 4: Shows some of the features that can be highlighted by calculating the minimum number of points needed to guarantee a playoff spot.

\begin{tabular}{|c|c|c|}
\hline Feature & Value & $\operatorname{Team}(\mathrm{s})$ \\
\hline Earliest day without guarantee & 64 days & St. Louis \\
\hline Most days without guarantee & 118 days & St. Louis \\
\hline Most times a team got lucky & 4 & Toronto, Boston, NY Rangers \\
\hline $\begin{array}{l}\text { Number of teams that got lucky } \\
\text { and earned a spot }\end{array}$ & 2 & NY Islanders, NY Rangers \\
\hline $\begin{array}{l}\text { Number of teams that got lucky } \\
\text { but failed to earn a spot }\end{array}$ & 7 & $\begin{array}{l}\text { Toronto, Boston, Washington, } \\
\text { Carolina, Edmonton, Phoenix, } \\
\text { Columbus }\end{array}$ \\
\hline
\end{tabular}

qualification and elimination problems for the NHL is presented. The calculation is efficiently computed by using a multi-stage solver that combines enumeration, flow network calculations and backtracking search.

This work represents the first complete and efficient solution to the NHL qualification and elimination problems. The key to scaling up the approach was a combination of enumeration, bounding, and constraint programming. As well, the costly work of calculating the actual division leaders was avoided as much as possible.

The 2005-06 and 2006-07 seasons were used to verify that our approach could solve realistic instances. Solving an instance only took a fraction of a second and each instance of the 2005-06 and 2006-07 seasons could be solved in several minutes. As well, clinching and elimination results could be announced as much as five days earlier than the Globe \& Mail (2005-2007).

A side effect of calculating the number of points needed to qualify for the playoffs is the ability to determine when the team is in danger of losing control of its destiny. These games, often described by coaches as "must win" games, can be identified as the loss reduces the maximum possible points to below the bound of the team. Nine different teams in the 2006-07 NHL season were identified that lost control of their fate and then gained that control back through mistakes by their opponents. Only two of these teams took full advantage of this situation and clinched a playoff spot. Of the nine teams that experienced this event, three of them experienced it four times. 
Table 5: The counts of problems in the 2007-08 season solved via the various stages of the solver. Positively solved instances means a solution was found and the bound must be increased. Negatively solved instances means that bound was valid for that instance. Any problem without a definitive solution was passed to the next phase of the solver.

\begin{tabular}{l|r|r} 
Solver Stage \& Result & $\begin{array}{c}\text { Number of } \\
\text { Instances (/5430) }\end{array}$ & $\begin{array}{r}\text { Cumulative } \\
\text { Percentage }\end{array}$ \\
\hline First Phase & 1212 & $22 \%$ \\
Second Phase (Positively) & 2249 & \\
Second Phase & 1524 & $92 \%$ \\
Third Phase (Positively) & 338 & \\
Third Phase (Negatively) & 107 & $100 \%$
\end{tabular}

\section{References}

Adler, I., Erera, A. L., Hochbaum, D. S., \& Olinick, E. V. (2002). Baseball, optimization and the world wide web. Interfaces, 32, 12-22.

Ahuja, R. K., Magnanti, T. L., \& Orlin, J. B. (1993). Network Flows: Theory, Algorithms and Applications. Prentice Hall.

Cheng, E., \& Steffy, D. (2008). Clinching and elimination of playoff berth in the NHL. International Journal of Operations Research, 5, 187-192.

Globe, \& Mail (2005-2007). Hockey scoreboard. Metro ed.

Gusfield, D., \& Martel, C. E. (2002). The structure and complexity of sports elimination numbers. Algorithmica, 32, 73-86.

ILOG S.A. (1998). ILOG Solver 4.2 user's manual.

Kern, W., \& Paulusma, D. (2004). The computational complexity of the elimination problem in generalized sports competitions. Discrete Optimization, 1, 205-214.

Lhomme, O. (2004). Arc-consistency filtering algorithms for logical combinations of constraints. In Proceedings of the 10th International Conference on the Principles and Practice of Constraint Programming (pp. 209-224). 
McCormick, S. T. (1999). Fast algorithms for parametric scheduling come from extensions to parametric maximum flow. Operations Research, 47, 744-756.

Ribeiro, C. C., \& Urrutia, S. (2005). An application of integer programming to playoff elimination in football championships. International Transactions in Operational Research, 12, 375-386.

Robinson, L. W. (1991). Baseball playoff eliminations: an application of linear programming. Operations Research Letters, 10, 67-74.

Rossi, F., van Beek, P., \& Walsh, T. (Eds.) (2006). The Handbook of Constraint Programming. Elsevier.

Russell, T., \& van Beek, P. (2008). Mathematically clinching a playoff spot in the NHL and the effect of scoring systems. In Proceedings of the 21st Conference of the Canadian Society for Computational Studies of Intelligence (pp. 234-245).

Russell, T., \& van Beek, P. (2009a). Determining the number of games needed to guarantee an NHL playoff spot. In Proceedings of the 6th International Conference on the Integration of $A I$ and $O R$ Techniques in CP for Combinatorial Optimization Problems (pp. 233-247).

Russell, T., \& van Beek, P. (2009b). Lessons learned from modelling the NHL qualification problem. In Proceedings of the Eighth International Workshop on Constraint Modelling and Reformulation (pp. 132-146).

Schulte, C., Tack, G., \& Lagerkvist, M. Z. (2011). Modeling and Programming with Gecode.

Schwartz, B. (1966). Possible winners in partially completed tournaments. SIAM Review, 8, 302-308.

Siek, J., Lee, L.-Q., \& Lumsdaine, A. (2001). Boost Graph Library: User Guide and Reference Manual. Addison-Wesley.

Van Hentenryck, P. (1989). Constraint Satisfaction in Logic Programming. MIT Press.

Wayne, K. D. (2001). A new property and a faster algorithm for baseball elimination. SIAM Journal on Discrete Mathematics, 14, 223-229. 